\title{
Vinculación de la ciudad histórico- turística de Puebla a través de itinerarios culturales
}

\author{
Patricia Domínguez Silva*
}

\begin{abstract}
Although having a vast cultural heritage, the city of Puebla in Mexico has a rather low visiting time among tourists. It seems that tourists that stopover in the Historic Centre of the city are not able to grasp their symbolic surroundings. This paper presents the evaluation results of two cultural itineraries in Puebla. We have analysed two journeys within the context of stops and phases in order to ponder the city's heritage. The results show that both journeys are unable to put in context the heritage of the places visited. In other words, in spite of their relevance to describe the cultural richness of the city, the visits do not fulfil their own objective: instruct, instil and educate. The greatest weakness of the journeys was found to be their lack of approach to urbanism, which is considered to be a characteristic element of cultural itineraries.
\end{abstract}

Keywords: historical-touristy city, itinerary, cultural tourism, Puebla.

\section{Resumen}

La ciudad Puebla, México, con un patrimonio cultural vasto, presenta debilidades en el tiempo de visita por parte de los turistas. Al parecer, el turismo que asiste al Centro Histórico no es capaz de percibir el contenedor simbólico que lo rodea. Este trabajo muestra los resultados de la evaluación de dos itinerarios culturales en Puebla, ambos se analizaron en el contexto de paradas y etapas para ponderar el patrimonio. Los resultados mostraron la falta de capacidad de ambos itinerarios para poner en contexto la riqueza patrimonial de los sitios visitados. Los itinerarios estudiados, relevantes para describir la riqueza patrimonial de la ciudad, distan de cumplir el objetivo de un itinerario: instruir, cultivar y letrar. La falta de aproximación al urbanismo detectada en estos itinerarios, teniendo en cuenta que el urbanismo es considerado un elemento característico e indiscutible de los itinerarios culturales, fue la mayor debilidad encontrada.

Palabras clave: ciudad histórico-turística, itinerario, turismo cultural, Puebla.

* Escuela de Negocios, Universidad de las Américas, Puebla. Correo-e: patricia.dominguez@udlap.mx. 


\section{Introducción}

Puebla se fundó en el siglo XVI, con el fin de instituir una ciudad para españoles en un lugar intermedio entre la Ciudad de México y el puerto de Veracruz. Los siglos XVII y XVIII fueron de bonanza económica y de gran esplendor de la vida urbana; durante ese periodo se definió la fisonomía urbana de la ciudad y sus características, apoyada, entre otros elementos, por un estilo de construcción regional que usa ladrillos, argamasas y azulejos para cubrir muros, torres y cúpulas.

Para poner en contexto la riqueza patrimonial de la ciudad histórica, su Zona Monumental e Histórica quedó constituida mediante decreto del año 1977 por 2,619 edificios correspondientes a 391 manzanas que comprenden la traza española, los barrios y los fuertes de Loreto y Guadalupe. En el año de 1987, el Centro Histórico ${ }^{1}$ fue inscrito en la lista del Patrimonio Cultural de la Humanidad de la Organización de las Naciones Unidas para la Educación, la Ciencia y la Cultura (UNESCO).

Las ciudades históricas se han convertido en un imán importante para el turismo, y la ciudad de Puebla no es la excepción. Se ha observado que en su centro histórico existen flujos importantes de turistas motivados principalmente por conocer el patrimonio cultural. Sabemos que el turista cultural se caracteriza por ser un viajero sensible a la calidad de la información que recibe, al entorno y al paisaje que él mismo elige visitar; busca en un lugar lo pintoresco, lo colorido y lo tradicional asociado a un ambiente de autenticidad, y en ocasiones se apoya en itinerarios turísticos no estructurados para conocer el lugar que visita. Como es comprensible, alrededor del Centro Histórico se han diseñado diversos itinerarios turísticos, algunos de ellos culturales, y es ahí donde surgen las siguientes interrogantes: ¿cómo se vincula el itinerario cultural con la ciudad histórica de Puebla? ¿Es posible, mediante los itinerarios culturales que se ofrecen, conocer la ciudad histórica?

La argumentación del trabajo parte de evaluar que las entidades responsables del quehacer turístico en la ciudad de Puebla confeccionan itinerarios turísticos que logran apuradamente relatar la ciudad histórica al turista con intereses culturales.

${ }^{1}$ Para efectos de este trabajo, se entiende como Centro Histórico la "parte de la ciudad que posee un alto valor estético e histórico como expresión de la cultura urbana y que se corresponde frecuentemente con las áreas histórico-monumentales de la urbe, las de mayor simbolismo y significación" (Zoido et al., 2000: 75). 
En la primera parte del escrito se expone un breve bosquejo referente al turismo cultural y la ciudad histórico-turística, así como una semblanza teórica sobre los itinerarios; posteriormente se introduce la problemática turística en relación con la ciudad histórico-turística de Puebla; a continuación se presentan y discuten los resultados de la investigación de campo que se llevó a cabo para obtener la información pertinente. Por último se registran, como conclusión, reflexiones sobre las observaciones del estudio.

\section{Turismo y su vinculación con la ciudad histórica por medio de itinerarios}

El turismo cultural es un fenómeno complejo, dado que el término cultura tiene diversas definiciones. No obstante, la globalización de los mercados, el incremento de las rentas y el tiempo libre en algunas sociedades han dado pauta al aumento en los viajes, fundamentalmente aquellos desplazamientos con contenido cultural. Este binomio turismo-cultura difícilmente puede soslayarse; es una realidad que ha propiciado que algunas ciudades, e incluso países, fundamenten en la cultura y en el patrimonio su atracción turística. Tal es el caso de Grecia y Egipto (Frohlich, 1993).

La literatura relacionada con el turismo cultural hace constar cómo en los últimos años se ha atribuido principalmente a la oferta cultural el crecimiento turístico de un destino (Domínguez y Bernard, 2001; Kerstetter, Confer y Graefe, 2001; Greffe, 2003; Castells, 2001a, 2001b; Fernández y Guzmán, 2001; Plaza, 2000). Llama la atención que en algunos casos este fenómeno ha dado pauta a que ciertos destinos turísticos traten de recupera el patrimonio cultural, y en otros casos se ha llegado al absurdo de poner en escena el bagaje cultural y patrimonial, en contra de la autenticidad del destino turístico (Ateljevic y Doorne, 2002; Goulding, 2000; Ryan, 2002; Human, 1999; Romero, 2001).

Esta manifestación ha implicado un replanteamiento en la gestión y desarrollo de sitios culturales, patrimoniales e históricos (Brooks, 1993). Sin embargo, y siguiendo a Santana (2003), ¿es realmente la cultura y el patrimonio lo que activa la demanda turística de un destino?

La bibliografía reseña el surgimiento de conflicto entre turistas y ciudadanos en destinos turísticos culturales como Chipre (Ayres, 2000) y el Triángulo Turístico de Sri Lanka (Amaraseke- 
ra y Navaratne, 1993). Siguiendo a Besculides, Lee y McCormick (2002), y a Aramberri (2001), la masificación del destino turístico ha obligado a revisar la relación entre la comunidad receptora y el turismo.

Por otra parte, las ciudades patrimoniales y sitios históricos han sufrido lo que Troitiño (2002) y Jansen-Verbeke (1998) han llamado "turistización". Este fenómeno ha propiciado que sitios histórico-patrimoniales se vean saturados de turistas, y que éstos hagan perder parte de la belleza que la ciudad ofrece. Por citar algunos casos, se mencionan Ámsterdam (Nijman, 1999), Bruselas (Dick, 2001), la Alhambra (García, 2001), Venecia (Russo, 2002) y París (Pearce, 1999).

\subsection{La ciudad histórica desde la perspectiva turística}

En términos generales podemos afirmar que la sociedad hoy en día es sobre todo urbana y posmoderna. Estas características le han brindado la pauta para disponer de mayor tiempo libre, lo que ha repercutido en un incremento en la actividad turística. ${ }^{2}$ Por otra parte encontramos que el turismo es fundamentalmente un evento urbano. Desde esta perspectiva, turismo y ciudad tienen una interrelación notable y permanente debido a que el turista vive la ciudad y se apropia de ella. Esta proximidad, que de manera introductoria se menciona en este ensayo, se basa en la premisa de que en las ciudades se localiza el foco principal del fenómeno turístico mundial. Tengamos presente que lo que se vende cuando el destino visitado es una ciudad es el producto turístico urbano.

Si consideramos a la ciudad histórica en particular y observamos en ella el hecho turístico, el fenómeno es complejo y ayuda a entender por qué no es fácil gestionar el turismo ni integrarlo a las estructuras urbanas, debido a que en la mayoría de los casos las ciudades históricas no estaban preparadas para esa función.

Uno de los problemas centrales, desde el punto de vista urbano, es cómo la ciudad histórica es convertida en recurso turístico. La ciudad turística es parte de la ciudad histórica donde los turistas se encuentran, conviven, comparten y compiten con los residentes y otros usuarios por diversos servicios, espacios y atracti-

\footnotetext{
${ }^{2}$ Por ejemplo, la Organización Mundial del Turismo calcula que más de 50\% de las llegadas corresponden a turismo urbano. También reporta que son las ciudades las que concentran la riqueza cultural (más de $70 \%$ de los activos patrimoniales en la Unión Europea).
} 
vos. En relación con este complejo maridaje, Ashworth y Tunbridge (1990) consideran que la ciudad histórica es dual: contiene un espacio histórico y a éste se le suma el espacio turístico.

De acuerdo con estas premisas, es importante determinar la relación entre turismo y ciudad histórica, de tal forma que puedan advertirse los vínculos que contribuyen a mantener una unión -aparentemente estrecha- de estos términos. Otro elemento que cobra relevancia es el hecho de que las ciudades históricas son espacios que registran un crecimiento interesante en cuanto a la afluencia turística; sin embargo, como lo señala Calle Vaquero (2002: 22), "no constituyen un espacio diseñado para el turismo ni la función turística ha constituido un elemento destacado en su configuración física y funcional actual”.

La ciudad histórico-turística se deriva de la articulación de dos categorías de turismo cultural: de interés especial y de lugar específico. El turista de interés especial está representado por quien, estando de vacaciones, tiene intereses semejantes a los que tiene en su vida cotidiana. Como ejemplo, un turista de interés especial que en su vida cotidiana está interesado en el trabajo de los pintores impresionistas, en su visita a París acudirá a observar obras pictóricas en el museo de Orsay; es por tanto un interés especial el que tiene para visitar este destino turístico. Para el turista de lugar específico, es el significado del lugar lo que lo estimula a visitar ese destino; el significado puede estar compuesto de diferentes atributos culturales previamente definidos. Ejemplificando este tipo de turista cultural, será aquel que haya definido previamente el consumo de algunas industrias culturales en particular, y que sumará en todo caso algunos valores estéticos y paisajísticos del lugar, entre otros significantes peculiares del sitio que visita. Sin embargo, este turista no acude a un espacio en específico para extender y ampliar el horizonte de intereses particulares que en su vida cotidiana practica; hará otro tipo de actividades, importantes para él, pero que no tienen una relación directa con las actividades e intereses de su vida habitual.

Cuando dirigimos estos tipos de turismo hacia la ciudad histórico-turística, no podemos encontrar una tipología única de turista. El turismo en las ciudades históricas corresponde a ambos tipos: tanto turistas con interés especial como de lugar específico.

Otra coyuntura de relevancia para la ciudad histórico-turística es la relación que guarda con el turismo urbano y el turismo cultural. Aunque el turismo, hasta cierto punto, existe en todas las ciudades, la importancia de su impacto varía no sólo con la magnitud 
del flujo de turismo sino más significativamente con el tipo de ciudad que recibe a ese turismo. Naturalmente hay muchas formas de turismo urbano y varias formas de turismo de ciudad. Como se mencionó con anterioridad, el turismo urbano se practica en las ciudades, las cuales atraen al turismo por diferentes motivos, ya sea compras (Miami, en Estados Unidos), actividades culturales (Londres, en Inglaterra), o por su patrimonio edificado (Morelia, en México), entre otros motivos. En cuanto al turista cultural, dependiendo del interés y siguiendo la taxonomía de Jansen-Verbeke, ${ }^{3}$ debemos considerar que los turistas tienen diferentes motivaciones culturales. Cabe mencionar que el empalme entre turismo en ciudades históricas -llamado también turismo patrimonialy turismo cultural es tan cerrado que frecuentemente estos términos son utilizados como sinónimos.

\subsection{Itinerarios}

El vocablo 'itinerario’ proviene del latín itinerarius, de iter, itíneris, camino, y se define como "descripción de un camino, indicando los lugares por donde se debe transitar". Boyra et al. (2003: 1) indican que proviene del griego dromológion, vocablo resultante de la combinación de dos palabras: drómos, camino, ruta, trayecto, y lógicos, instruir, cultivar, letrar.

En términos generales, los itinerarios tienen como objetivo cubrir una cierta distancia en el territorio mediante una sucesión de etapas y paradas con una finalidad determinada. Las etapas y las paradas son los elementos principales que estructuran un itinerario utilizando y ocupando el territorio de una manera concreta. Las paradas son obligadas, pues permiten efectuar la visita a cada uno de los recursos específicos que conforman el itinerario, con la premisa de que el conjunto de visitas es el motivo principal para recorrer el itinerario. Las etapas entre las paradas permiten al turista contextualizar el conjunto de recursos que se visitan en un territorio (Boyra et al., 2003).

Dado que teóricamente se parte del supuesto de que el itinerario permite visitar y poner en contexto los recursos dentro del territorio, las etapas deben fungir como vínculos entre las paradas -los lugares visitados- y permitir al momento descubrir y conocer la ciudad. Como lo afirman Boyra et al. (2003: 4), "la

${ }^{3}$ Jansen-Verbeke (1998) realiza una segmentación de los visitantes en relación con la cultura: turistas de motivación cultural, turistas de inspiración cultural y turistas atraídos por la cultura. 
aproximación al urbanismo es por lo tanto un elemento característico e indiscutible de los itinerarios culturales de la ciudad”.

\section{Puebla, ciudad turística}

Para México, y según datos del estudio estratégico de viabilidad del turismo cultural del Centro de Estudios Superiores en Turismo (Cestur, 2002), la cultura ocupa el sexto lugar en cuanto a motivación principal para el turismo nacional y el cuarto para el turismo internacional. El turismo motivado particularmente por la cultura representa $5.5 \%$ entre los viajeros nacionales y $3 \%$ entre los internacionales. Los turistas que manifestaron la realización de su viaje por otros motivos y que tuvieron actividades relacionadas con la cultura representaron $37 \%$ de los visitantes internacionales y $35 \%$ de los nacionales. Estas cifras permiten al sector turístico calcular que el flujo de turistas que desarrollan actividades culturales representa alrededor de 40\% del volumen total de los turistas que viajan a México.

La ciudad de Puebla, capital del estado de Puebla y cabecera del municipio del mismo nombre, está situada al este de la Ciudad de México. Fue fundada en el valle de Cuetlaxcoapan hacia el primer tercio del siglo XVI. La fecha de la fundación se refiere al 16 de abril de 1531. Esta ciudad fue concebida como modelo urbanístico en su época.

La ciudad de Puebla ha sido destino turístico de rasgos culturales desde tiempos pasados. Montero (2002: 14) comenta: “[...] desde el año de 1876 Puebla fue una de las ciudades más visitadas por los turistas, porque en ella se recreaba la vistosidad de sus edificios y casonas antiguas, además de sus centros ceremoniales prehispánicos como los de Cholula y Manzanilla”.

La ciudad histórica tiene una extensión de $6.99 \mathrm{~km}^{2}$. En cuanto a su población, Álvarez Mora (2001: 64) comenta que para el año de 1978, la población del Centro Histórico era de 350,000 mil habitantes; para 1990, esa población había disminuido hasta llegar a los 86,059 habitantes, y reporta para 1995 una población de 81,706 habitantes; "en la actualidad nos encontramos con una población de 69,162 habitantes".

Por su cercanía con la capital del país, Puebla es fundamentalmente un destino de negocios. Para el año 2004, y según datos de la Unidad de Desarrollo Administrativo, Planeación e Informática (2004), la ocupación hotelera fue de 64.49\%; la derrama económica, de $\$ 3,796,731,790$, y la afluencia de visitantes, de 
4,039,519. La estadía promedio fue de 1.62 días. El promedio de edad de los visitantes fue de 35 años, $84.49 \%$ fueron nacionales y $15.51 \%$ extranjeros. Las estadísticas publicadas por la Secretaría de Turismo estatal indican que los turistas nacionales que permanecen menos de un día en la ciudad en promedio la recorren en 10 horas, mientras que los extranjeros en cinco. Los turistas nacionales y extranjeros que la visitan por más de un día permanecen de uno a dos días en promedio.

Puebla ha estado constantemente presente en el desarrollo turístico de México, y se ha colocado como destino turístico en diferentes programas, como el de Ciudades Coloniales y la Ruta de los Dioses, entre otros. Sin embargo, y a pesar de los esfuerzos de promoción, los datos de afluencia turística no apuntan a que Puebla sea un sitio turístico destacado en el mapa de destinos turísticos culturales. Para sustentar esta afirmación, nótense los últimos datos disponibles: en el estudio Expectativas de viaje del turismo doméstico para las vacaciones de verano 2003, llevado a cabo por el Cestur en los principales mercados emisores de México, en el conjunto de "Destinos ideales" o top of mind, Puebla no figuró en ninguno de los casos; para la variable "destinos planeados", nuevamente no fue mencionada. Estas consideraciones permiten poner en contexto el problema de esta ciudad: un Centro Histórico declarado por la UNESCO como Patrimonio Cultural de la Humanidad, diversas incursiones de este sitio dentro de la planificación turística de México, un débil posicionamiento en el mercado como destino vacacional y una estadía corta de los visitantes.

En cuanto a los itinerarios turísticos, la ciudad de Puebla está contenida en las principales guías turísticas nacionales y extranjeras. En las páginas oficiales de promoción turística federal, estatal y municipal, se proporciona información sobre atractivos turísticos y diversos itinerarios de la ciudad.

En los siguientes párrafos, se describen los itinerarios propuestos por la Secretaría de Turismo estatal y por la Dirección de Turismo municipal.

\subsection{Secretaría de Turismo estatal}

La Secretaría de Turismo ofrece cuatro programas turísticos de carácter cultural: Sitios Arqueológicos, Bellezas Arquitectónicas, Ciudades Turísticas y Rutas Turísticas. A efectos de este trabajo interesa el programa de Bellezas Arquitectónicas, el cual 
considera la visita al Centro Histórico y a 16 edificios entre iglesias, conventos y ex haciendas ubicados alrededor de la ciudad de Puebla. En el sitio web de información turística del estado (Consejo Estatal de Promoción Turística, 2003) se encuentran los siguientes itinerarios turísticos: 1. Angelópolis, 2. Sierra Mágica, 3. Sierra Mixteca, 4. Centro Histórico. En el itinerario correspondiente al Centro Histórico se ofrecen cinco opciones de recorridos dependiendo del tiempo de estancia. Es preciso indicar en este momento que las cinco opciones de recorrido ponen énfasis en el Centro Histórico y los alrededores de la ciudad de Puebla.

\subsection{Dirección de Turismo municipal}

La Dirección de Turismo municipal tiene tres propuestas de recorridos ofrecidos de manera gratuita, sólo se requiere hacer reservación. Cabe mencionar que todos los recorridos están abocados al Centro Histórico. Son los siguientes:

1. Recorrido del Centro Histórico: con dos opciones de recorridos. La opción "a” considera 10 visitas que incluyen tres espacios públicos: un museo, un edificio y cuatro iglesias. La opción "b" comprende siete visitas con tres espacios públicos: un museo, dos edificios y una iglesia.

2. Recorrido conventual: incluye la visita a cinco iglesias conventuales.

3. Recorrido de patios coloniales: con dos opciones de recorridos, cada uno de ellos visita ocho patios coloniales.

\section{Evidencia empírica: itinerarios culturales en la ciudad histórico-turística de Puebla}

Partimos de la premisa de que los viajeros que conciernen a este estudio, aquellos con intereses culturales, siguen un itinerario que cubre necesidades y valores epistemológicos. Para apreciar los itinerarios culturales en su conformación de paradas y etapas y evaluar su capacidad para que el visitante pueda leer e interpretar el contenido simbólico de la ciudad histórico-turística, fue necesario en un primer momento de la investigación definir cuáles serían las fuentes de información. Se consideró clasificar las guías turísticas en dos grandes bloques: 
1. Las elaboradas por las instituciones gubernamentales en los ámbitos estatal y municipal (Secretaría de Turismo estatal y Dirección de Turismo municipal).

2. Las generadas por organizaciones privadas.

En este trabajo se presentan los resultados de un itinerario elaborado por la Secretaría de Turismo estatal, y de otro elaborado por la Dirección de Turismo municipal. A continuación se presentan los resultados de la evaluación de esos dos itinerarios culturales.

\subsection{Secretaría de Turismo del estado: Recorrido 3}

De los recorridos ofrecidos por la Secretaría de Turismo del estado, nos inclinamos por aquellos que se concentraban en el Centro Histórico de la ciudad de Puebla. Los itinerarios brindan información concreta sobre los atractivos y muestran un tiempo aproximado de recorrido, pero no hacen referencia al medio de transporte en la página web del Consejo de Promoción Turística. De esos paseos al Centro Histórico se seleccionó el Recorrido 3. El sitio web donde se obtuvo la información ha sido modificado. ${ }^{4} \mathrm{Se}$ consideró oportuno seleccionar este recorrido porque ofrece la oportunidad de integrar dos conventos importantes en la historia conventual y gastronómica de la ciudad: el ex convento de Santa Rosa y el ex convento de Santa Mónica. Una razón adicional para esta selección fue la oportunidad que da para conocer la zona norte-poniente del Centro Histórico. Para efectuar la evaluación del itinerario se consideró que en las etapas que comprendieran más de cinco manzanas se haría el recorrido en auto, y en las etapas menores de cinco manzanas se haría el recorrido andando.

\subsubsection{Paradas y etapas}

Debido a que este recorrido cubre grandes distancias, fue un elemento que se juzgó significativo en la evolución: se consideró relevante comprobar si en el tiempo sugerido se alcanzaba efectivamente a cubrir todo el itinerario. Otro elemento fue la capacidad que la guía colocada en el sitio web tiene para ubicar espacialmente al visitante. Un aspecto adicional y fundamental en la evaluación de este recorrido fue la capacidad descriptiva del iti-

${ }^{4}$ http://www.turismopuebla.com.mx/5opciones.htm\#recorrido3. 
nerario en función de las etapas que se recorrían. Tómese en consideración que "la aproximación al urbanismo es por lo tanto un elemento característico e indiscutible de los itinerarios culturales de la ciudad" (Boyra et al., 2003: 1).

Las paradas indicadas en este recorrido son las siguientes:

1. Museo Nacional del Ferrocarril (11 Norte y 12 Poniente).

2. Museo y Pinacoteca José Luis Bello y Zetina (5 de Mayo y 6 Oriente).

3. Ex convento de Santa Mónica (18 Poniente 103).

4. Ex convento de Santa Rosa (14 Poniente y 3 Norte).

5. Museo de la No Intervención (Fuerte de Loreto).

6. Fuertes de Loreto y Guadalupe (Centro Cívico 5 de Mayo).

7. Mercado de Antigüedades "Plazuela de los Sapos" (5 Oriente y 6 Sur).

El tiempo previsto de recorrido, según esta guía turística, es de un día (cinco horas en la mañana y tres horas en la tarde). Cabe hacer notar que los museos abren a las 10:00 y cierran a las 16:30 horas. El recorrido se inició a las 10:00 y fue finalizado a las 17:00 horas. En ese tiempo, las paradas que pudieron visitarse fueron las siguientes:

1. Museo Nacional del Ferrocarril (11 Norte y 12 Poniente).

2. Museo y Pinacoteca José Luis Bello y Zetina (5 de Mayo y 6 Oriente).

3. Ex convento de Santa Rosa (14 Poniente y 3 Norte).

4. Ex convento de Santa Mónica (18 Poniente 103).

Como puede observarse, fue modificada la secuencia de una parada: se visitó en primer término Santa Rosa y posteriormente Santa Mónica, pues recorrer el trayecto a pie resultó más lógico para el desplazamiento.

\subsubsection{Etapas}

La evaluación de las etapas fue infortunada. El recorrido que vincula las paradas, sin sugerencia de la guía turística de por dónde transitar, nos condujo a caminar por calles que si bien son interesantes desde la perspectiva arquitectónica y urbanística, al carecer de información sobre estos componentes, resultó un camino complejo. Sin información ni señalización turística en esa parte 
del Centro Histórico, llegar a los sitos de destino resultó una verdadera hazaña. No hay indicaciones sobre los museos, ni caseta de información turística, o cualquier otro tipo de auxilio turístico que ayude al visitante a llegar del Museo José Luis Bello y Zetina al ex convento de Santa Rosa. Se transita por la calle 5 de Mayo, la cual resulta agresiva para los turistas, de tal forma que a partir del jardín de San Luis es prácticamente imposible encontrar turistas caminando, pues la inseguridad se percibe en el ambiente. El deterioro de la imagen urbana que se nota en esa área del Centro Histórico es importante, como la falta de mantenimiento al mobiliario urbano. La deficiente limpieza en las calles adyacentes al mercado 5 de Mayo hace que la percepción positiva sobre la ciudad decaiga.

Continuando hacia el ex convento de Santa Mónica, nuevamente éste pudo ser identificado por seguir la numeración de las casas en la calle, pues no hay una señal clara y visible sobre este importante inmueble. Para llegar allí se transitó por interesantes calles que concentran oficios típicos de la ciudad de Puebla, como la alfarería, la producción de talavera y la hojalatería, que produce los típicos anafres para la elaboración de "chalupas" y "molotes", entre otros alimentos típicos. También se transita por establecimientos que trabajan la jarcería. Es justo destacar que por esas calles se encuentran antiguas panaderías, tan características de la ciudad de Puebla, donde se produce de manera artesanal el "pan de dulce" y donde la imaginación del panadero se deja ver confeccionando las famosas conchas de chocolate y vainilla, las "chilindrinas" y el pan de manteca, entre otros. En síntesis, las calles que unen los recorridos entre el Museo José Luis Bello y Zetina y los ex conventos de Santa Rosa y Santa Mónica presentan a la Puebla tradicional, probablemente olvidada, pero que aún en sus calles muestra artes y oficios que se iniciaron desde la Colonia. Sin embargo, estos entramados que hacen que el visitante se conecte con la ciudad no se mencionan en la guía turística.

\subsubsection{Conclusiones sobre el Recorrido 3}

Este itinerario cultural, importante para descubrir la vida conventual de la ciudad y, a través de sus calles, la traza de la ciudad colonial, dista mucho de cumplir el objetivo de un itinerario: ilustrar, letrar. Las distancias sugeridas son amplias, lo que implica recorrer un tramo andando y el otro en taxi o automóvil, pues no hay un servicio de transporte público seguro que pueda 
satisfacer al turista en general. Este itinerario implica cruzar la ciudad de oeste a este, dado que se inicia en el Museo Nacional del Ferrocarril, el cual se encuentra en las calles 11 Norte y 12 Poniente, y se finaliza en los Fuertes de Loreto y Guadalupe (Centro Cívico 5 de Mayo), ubicados en el lado opuesto del museo. Debido a que los museos cierran a las 16:30 horas, en el recorrido sólo pudimos visitar tres museos, nos vimos imposibilitados para llegar al Museo de la No Intervención, por lo que se decidió dar fin al recorrido a la salida del ex convento de Santa Mónica.

\subsection{Dirección de Turismo municipal: itinerario conventual}

Los itinerarios ofrecidos por la Dirección de Turismo municipal, al contar con la orientación de guías turísticos, están sujetos a la calidad interpretativa de estos trabajadores. Debido a que el interés de este estudio radica en los itinerarios culturales, se seleccionó el itinerario conventual, pues se consideró que no sólo refleja la rica historia virreinal de la ciudad, sino que también pone de manifiesto la riqueza arquitectónica de la ciudad históricoturística. También se asumió, dado que el itinerario se realizaría andando, que la valoración de paradas y etapas podría hacerse con una mayor objetividad. El grupo de visitantes quedó constituido por cinco personas; se juzgó relevante invitar a una persona experta en arte colonial poblano, para tener al final del recorrido una opinión calificada sobre la calidad interpretativa del guía y conocer si algún elemento importante había sido omitido. Cabe aclarar que ningún miembro del grupo conocía la finalidad de realizar el recorrido; simplemente se les invitó a "un paseo por el centro de Puebla”.

\subsubsection{Paradas y etapas}

La evaluación de este itinerario cultural se basó en la capacidad, por medio del guía, para poner en contexto los recursos en el área delimitada en el recorrido, y así apreciar si las etapas verdaderamente fungían como un elemento de unión entre los lugares visitados (paradas), y sí efectivamente permitían conocer esa parte del Centro Histórico a través de sus calles.

En el folleto informativo, el itinerario está constituido por seis paradas y comienza en la Dirección de Turismo. A continuación se enuncia el contenido de este recorrido: 
1. Dirección de Turismo municipal (Portal Hidalgo 14).

2. Templo y ex convento de la Concepción (avenida 7 Poniente esquina calle 16 de Septiembre).

3. Templo y ex convento de Santa Inés (avenida 9 Oriente esquina calle 3 Sur).

4. Templo y convento de las Capuchinas (avenida 9 Oriente esquina calle 16 de Septiembre).

5. Templo y ex convento de la Soledad (calle 2 Sur esquina avenida 13 Oriente).

6. Templo y ex convento Iglesia de San Jerónimo (avenida 7 Oriente esquina calle 3 Sur).

No obstante, el recorrido fue modificado por el guía; quitó conventos -La Concepción, Santa Inés- y añadió el templo del Espíritu Santo. Como puede inferirse, la temática del itinerario se perdió.

\subsubsection{Paradas}

Las paradas antes mencionadas fueron visitadas. Sin embargo, la narración del guía estuvo enfocada invariablemente al estilo arquitectónico de las iglesias. Hubo escasas menciones a los conventos y se omitió información sobre su fundación y si estaban o no en servicio. Los datos anecdóticos, siempre de interés para el turista, fueron obviados.

\subsubsection{Etapas}

Las etapas son el punto focal para la interpretación de la ciudad, pues vinculan la parada con el territorio; entonces teóricamente debe existir información notable en torno al territorio visitado. Estos datos facilitan al visitante una comprensión del territorio; en este caso, del Centro Histórico en su ruta conventual. Por lo tanto, conocer de sus calles, de la historia e historias de esas calles, de sus casas y habitantes hace que el turista comprenda la ciudad, y que de alguna manera plasme en su mente el mapa del recorrido, que finalmente formará parte de sus recuerdos de viaje; podrá decir: he conocido la ciudad. ¿De qué sirve comentar que se conoce un hito en alguna ciudad histórica, si no se recuerda dónde está y por qué está? Las etapas tejen realmente el itinerario cultural, son las que graban la parada en la mente y por su mediación el entramado urbanístico cobra sentido para el viaje- 
ro. En el itinerario que se recorrió, las etapas fueron infortunadas en contenido.

\subsubsection{Conclusiones sobre el itinerario conventual}

El recorrido terminó a una calle del lugar de origen del itinerario. Esto evitó recorrer parte de una de las avenidas más simbólicas del Centro Histórico, la de Juan de Palafox y Mendoza, donde, nuevamente, el turista puede apreciar bellos edificios con hermosos patios, todos plenos de historia, como la Casa Arronte (avenida Juan de Palafox y Mendoza 219), que actualmente funciona como la Biblioteca de la Benemérita Universidad Autónoma de Puebla (BuAP); y la Casa Presno (avenida Juan de Palafox y Mendoza 208), hoy Instituto de Ciencias Sociales y Humanidades de la BUAP, por mencionar sólo dos de las espléndidas edificaciones con que cuenta esta avenida.

Si bien el tema del itinerario aquí estudiado fue el de los conventos, o, propiamente dicho, "las iglesias de los conventos", la interpretación de la ciudad mediante de este itinerario no debe omitir los elementos que acompañan la historia conventual y las calles por las cuales se transita, pues son bases que perfilan a la ciudad. En este sentido, las etapas son fundamentales como fuente interpretativa de lo que se ha visitado. Sin estos vínculos, el itinerario queda trunco; difícilmente el turista podrá conocer y reconstruir la ciudad a partir de las paradas.

Reparando en lo que sucedía a medida que se avanzaba en el itinerario conventual, se observó que por esas calles no transitan grupos de turistas, y que más bien deambula por ellas uno que otro turista extranjero, con su guía turística bajo el brazo y con el rostro desencajado. Las preguntas aún quedan en el aire: ¿̇cómo conectarán con la ciudad?, ¿̇lograrán finalmente su objetivo, conocer algo de la ciudad, de su historia?

Si se retoma el significado de itinerario como ruta que instruye, consideramos que en esta ocasión el itinerario cultural conventual se ha quedado al margen de la ciudad histórica de Puebla.

\section{Conclusión}

La ciudad de Puebla, con el patrimonio cultural contenido en su Centro Histórico, está en condiciones de ser un destino turístico importante, sobre todo para el turismo nacional. En ocasiones se considera que contar con atractivos turísticos o una adecuada 
promoción del destino es suficiente para aumentar los flujos turísticos, y no se toma en cuenta que son diferentes factores los que hacen al destino atractivo. La ciudad histórico-turística de Puebla sigue teniendo una belleza virreinal como pocas ciudades en América Latina debido, entre otras causas, a la falta de interés durante años en este importante contenedor simbólico, lo que conservó la belleza de sus edificaciones. Sin embargo, la ciudad histórico-turística, como se ha apuntado en este artículo, no fue diseñada para esta función, y, al parecer, se requiere avanzar en la interpretación de la ciudad; probablemente el primer paso sea la confección acertada de itinerarios culturales que permitan entenderla correctamente. Por lo revisado en este trabajo, puede afirmarse que el turista difícilmente logra, con base en los itinerarios, conocer la ciudad histórica, debido, entre muchos otros factores, a la desvinculación entre el itinerario cultural y la ciudad histórico-turística. La falta de aproximación al urbanismo, el cual es considerado un elemento característico e indiscutible de los itinerarios culturales, fue la mayor debilidad detectada en las paradas y etapas analizadas en estos itinerarios.

Por supuesto que hay más problemas de los aquí expuestos, y que por motivos de espacio no son discutidos: calidad de las guías turísticas, de los servicios de información y señalización efectiva para el turista, entre otros, que ayuden a explicar de manera precisa la razón por la cual el turista que visita la ciudad tiene una estadía corta.

Sin embargo, las pautas para el mejoramiento de la calidad interpretativa para el turista que visita esta ciudad pueden establecerse con la confección de itinerarios que vinculen historia, leyenda, tradición, arquitectura y urbanismo, todo ello en un ambiente de autenticidad.

Por otra parte, se considera que esta ciudad histórico-turística tiene más matices que los explorados hasta ahora. Pueden generarse itinerarios que se dirijan a los diferentes tipos de visitantes con intereses culturales precisos. Así, podemos distinguir contenidos temáticos que narren la vida conventual, las monjas coronadas, los sonidos de Puebla, las puertas de Puebla, los balcones esquinados, entre otros muchos temas. 


\section{Bibliografía}

Álvarez Mora, Alfonso (2001), "Reforma urbanística o recreación monumental: un dilema abierto para el Centro Histórico de Puebla”, en Mireia Viladevall I. Guasch (coord.), Ciudad, patrimonio y gestión, Benemérita Universidad Autónoma de Puebla, Puebla.

Amarasekera, Athula y Dayapriya Navaratne (1993), "Cultural Tourism and Visitor Effects on the Local Population with Special Reference to the Cultural Triangle of Sri Lanka”, Cultural Tourism, International Scientific Symposium, 10th General Assembly, Icomos (International Council on Monuments and Sites), pp. 1-4.

Aramberri, Julio (2001), “The Host Should Get Lost. Paradigms in the Tourism Theory", Annals of Tourism Research, Elsevier Science, 28 (3): 738-761.

Ashworth, Gregory J. y John E. Tunbridge (1990), The TouristicHistoric City, Belhaven Press, Londres.

Ateljevic, Irena y Stepen Doorne (2002), "Representing New Zealand Tourism Imagery and Ideology", Annals of Tourism Research, Elsevier Science, 29 (3): 648-667.

Ayres, Ron (2000), "Tourism as a Passport to Development in Small States: Reflection on Cyprus”, International Journal of Social Economics, Emerald Group Publishing Limited, 27 (2): 114-133.

Besculides, Antonia, Martha E. Lee y Peter J. McCormick (2002), "Resident's Perceptions of the Cultural Benefits of Tourism”, Annals of Tourism Research, Elsevier Science, 29 (2): 303-319.

Boyra, Josep, Maria Josep Conde e Inmaculada de Solà-Morales (2003), Els itineraris culturals a la ciutat de Barcelona l'any 2002, ponencia presentada en el XII Simposio Internacional de Turismo y Ocio, http://www.esade.es/cedit2003/ pdfs/boyrajose.pdf, 24 de mayo. 
Brooks, Graham (1993), "Visitation to Major Heritage Sites Some Essential Planning Considerations", Cultural Tourism, International Scientific Symposium, $10^{\text {th }}$ General Assembly, Icomos, pp. 14-19.

Calle Vaquero, Manuel de la (2002), La ciudad histórica como destino turístico, Ariel, Barcelona.

Castells, Margalida (2001a), La interpretación del patrimonio como estrategia de desarrollo territorial: reflexiones a propósito de la interpretación de la Basílica Paleocristiana de Son Peretó (Manacor, Mallorca, España), ponencia presentada en el Congreso Virtual de Turismo, http://www.naya. org.ar/turismo_cultural/congreso/ponencias/margalida_ castells.htm, $2 \overline{3}$ de mayo de 2003.

(2001b) Reencontrar el patrimonio. Estrategias de desarrollo territorial a partir de la interpretación, comunicación presentada en Congreso Virtual de Turismo, http:// www.naya.org.ar/turismo/congreso/ponencias/margalida_ castells.html, 23 de mayo de 2003.

Cestur (Centro de Estudios Superiores en Turismo) (2002), Turismo cultural, Cestur, México.

Consejo Estatal de Promoción Turística (2003), Turismo en Puebla, http://www.turismopuebla.com.mx, 23 de julio.

Dick, Leonard (2001), "Brussels: Belgium Doubles its World Heritage Sites", Europe, abril, 405: 38-39.

Domínguez, Patricia y Alicia Bernard (2001), “La interpretación del patrimonio como estrategia de conservación, aprovechamiento y desarrollo en Santa María Tonanzintla”, en Daniel Meyer (ed.), Turismo y desarrollo sostenible, Universidad Externado de Colombia, Medellín, pp. 41-53.

Fernández, Guillermina y Aldo Guzmán (2001), El patrimonio bistórico-cultural revalorizado en el marco de un desarrollo sustentable del turismo, ponencia presentada en el Congreso Virtual de Turismo, http://www.naya.org.ar/ 
turismo_cultural/congreso/ponencias/aldo_ramos.htm, 23 de mayo de 2003.

Frohlich, Martin (1993), "Is There a Tourist Culture? Cultural Tourism”, Cultural Tourism, International Scientific Symposium, $10^{\text {th }}$. General Assembly, Icomos, pp. 66-69.

García Hernández, María (2001), “Capacidad de acogida turística y gestión de flujos de visitantes en conjuntos monumentales: el caso de La Alhambra", dossier 11: Turismo en ciudades históricas, boletín PH, 36, septiembre, Junta de Andalucía, http:/www.juntadeandalucia.es/cultura/ iaph/publicaciones/dossiers/dossier11/dossier11art4.htm, 23 de mayo 2004.

Goulding, Christina (2000), The Commodification of the Past, Postmodeern Pastiche, and the Search for Authentic Experiences at Contemporary Heritage Attractions", European Journal of Marketing, Emeral, 34 (7): 835-853.

Greffe, Xavier (2003), “¿Es el patrimonio un incentivo para el desarrollo?", dossier 14: El patrimonio como factor de desarrollo: balance y perspectivas, boletín $\mathrm{PH}, 42$, febrero, Junta de Andalucía, http://www.juntadeandalucia.es/ cultura/iaph/publicaciones/dossiers/dossier14/dossier14art1. html, 20 de marzo de 2003.

Human, Brian (1999), "Kodachrome Icons: Photography, Place and the Theft of Identity", International Journal of Contemporary Hospitality Management, Emereald Group Publishing Limited, 11 (2-3): 80-84.

Jansen-Verbeke, Myriam (1998), "Tourismification of Historical Cities", Annals of Tourism Research, Elsevier Science, 25 (3): 739-741.

Kerstetter, Deborah, John Confer y Alan R. Graefe (2001), “An Exploration of the Specialization Concept within the Context of Heritage Tourism", Journal of Travel Research, Sage Publications, Inc., 39 (3): 267-274. 
Montero Pantoja, Carlos (2002), Colonias de Puebla, Benemérita Universidad Autónoma de Puebla-Museo Amparo, Puebla.

Nijman, Jan (1999), "Cultural Globalization and the Identity of Place: The Reconstruction of Amsterdam", Ecumene, Hodder Arnold Journals, 6 (2): 146-164.

Pearce, Douglas (1999), "Tourism in Paris. Studies at the Microscale", Annals of Tourism Research, Elsevier Science, 26 (1): 77-97.

Plaza, Beatriz (2000). "Evaluating the Influence of a Large Cultural Artifact in the Attraction of Tourism: The Guggenheim Museum Bilbao Case", Urban Affairs Review, 36 (2), 264-276.

Romero Moragas, Carlos (2001), "Ciudad, cultural y turismo: calidad y autenticidad", dossier 11: Turismo en ciudades históricas, boletín PH, 36, septiembre, Junta de Andalucía, http:// www.juntadeandalucia.es/cultura/iaph/publicaciones/ dossiers/dossier11/dossier11art1.htm, 21 de marzo de 2003.

Russo, Antonio Paolo (2002), “The 'Vicious Circle' of Tourism Development in the Heritage Cities", Annals of Tourism Research, Elsevier Science, 29 (1): 165-182.

Ryan, Christopher (2002), “Tourism and Cultural Proximity. Examples from New Zealand", Annals of Tourism Research, Elsevier Science, 29 (4): 952-971.

Santana, Agustín (2003), "Patrimonios culturales y turistas: unos leen lo que otros miran", Pasos. Revista de Turismo y Patrimonio Cultural, 1 (1): 1-12.

Troitiño Vinuesa, Miguel Ángel (2002), “Ciudades españolas Patrimonio de la Humanidad: desafíos de interpretación y gestión”, dossier 13: Patrimonio mundial 1972-2002, boletín PH, 40/41, noviembre, Junta de Andalucía, http:// www.juntadeandalucia.es/cultura/iaph/publicaciones/ dossiers/dossier13/dossier13art3.htm, 20 de mayo de 2005. 
Unidad de Desarrollo Administrativo, Planeación e Informática (2004), Indicadores Estadísticos de la Actividad Turística en Puebla, archivo de datos, Secretaría de Turismo de Puebla, Puebla.

Zoido Naranjo, Florencio et al. (2000). Diccionario de geografía urbana, urbanismo y ordenación del territorio, Ariel, Ariel Referencia, Barcelona.

Recibido: 8 de diciembre de 2004. Reenviado: 25 de mayo de 2005. Aceptado: 20 de junio de 2005.

Patricia Domínguez Silva tiene el doctorado en urbanística. Actualmente está adscrita a la Escuela de Negocios de la Universidad de las Américas, en Puebla. Sus líneas de investigación se orientan al turismo urbano y el turismo cultural. Entre sus publicaciones se cuentan: "Cómo conseguir una ciudad más habitable y competitiva", preámbulo a la Carta de Puebla I, Honorable Ayuntamiento de Puebla, Puebla, México, 2003; con Alicia Bernard y Raúl Valdés, 'Turismo y patrimonio en el siglo xxi', capítulo 1 "Patrimonio y turismo. Reconversión del patrimonio en hoteles", Ex convento de la Concepción y Mesón Sacristía de la Compañia, Centro de Investigaciones y Estudios Turísticos, Puebla, México; también con Alicia Bernard, "Gastronomía, la herencia olvidada. Patrimonio Cultural y Turismo", Cuadernos 3, Pensamientos acerca del patrimonio cultural. Antología de textos, Conaculta, México, 2003, pp. 261-306, y Tourism and Heritage Relationships: Global, National and Local Perspectives Gastronomy, the Neglected Heritage, The Center for Travel \& Tourism. Ha colaborado con diferentes grupos de investigación nacionales e internacionales en otras instituciones. 